\title{
Existence of homoclinic solutions for a class of difference systems involving $p$-Laplacian
}

\section{Qiongfen Zhang*}

\section{"Correspondence:}

qfzhangcsu@163.com

College of Science, Guilin University

of Technology, Guilin, Guangxi

541004, P.R. China

\begin{abstract}
By using the critical point theory, some existence criteria are established which guarantee that the difference $p$-Laplacian systems of the form $\Delta\left(|\Delta u(n-1)|^{p-2} \Delta u(n-1)\right)-a(n)|u(n)|^{q-p} u(n)+\nabla W(n, u(n))=0$ have at least one or infinitely many homoclinic solutions, where $1<p<(q+2) / 2, q\rangle 2, n \in \mathbb{Z}, u \in \mathbb{R}^{N}$, $a: \mathbb{Z} \rightarrow(0,+\infty)$, and $W: \mathbb{Z} \times \mathbb{R}^{N} \rightarrow \mathbb{R}$ are not periodic in $n$.

MSC: $34 C 37 ; 35 A 15 ; 37 J 45 ; 47 J 30$
\end{abstract}

Keywords: homoclinic solutions; variational methods; difference $p$-Laplacian systems

\section{Introduction}

Consider homoclinic solutions of the following $p$-Laplacian system:

$$
\Delta\left(|\Delta u(n-1)|^{p-2} \Delta u(n-1)\right)-a(n)|u(n)|^{q-p} u(n)+\nabla W(n, u(n))=0, \quad n \in \mathbb{Z},
$$

where $1<p<(q+2) / 2, q>2, n \in \mathbb{Z}, u \in \mathbb{R}^{N}, a: \mathbb{Z} \rightarrow(0,+\infty)$, and $W: \mathbb{Z} \times \mathbb{R}^{N} \rightarrow \mathbb{R}$ are not periodic in $n . \Delta$ is the forward difference operator defined by $\Delta u(n)=u(n+1)-u(n)$, $\Delta^{2} u(n)=\Delta(\Delta u(n))$. As usual, we say that a solution $u$ of $(1.1)$ is homoclinic (to 0$)$ if $u(n) \rightarrow$ 0 as $n \rightarrow \pm \infty$. In addition, if $u(n) \not \equiv 0$, then $u(n)$ is called a nontrivial homoclinic solution. We may think of (1.1) being a discrete analogue of the following differential system:

$$
\frac{d}{d t}\left(|\dot{u}(t)|^{p-2} \dot{u}(t)\right)-a(t)|u(t)|^{q-p} u(t)+\nabla W(t, u(t))=0, \quad t \in \mathbb{R}
$$

When $p=2$, (1.1) can be regarded as a discrete analogue of the following second-order Hamiltonian system:

$$
\ddot{u}(t)-a(t)|u(t)|^{q-2} u(t)+\nabla W(t, u(t))=0, \quad t \in \mathbb{R} .
$$

Problem (1.2) has been studied by Shi et al. in [1] and problem (1.3) has been studied in [2-4]. It is well known that the existence of homoclinic orbits for Hamiltonian systems is a classical problem and its importance in the study of the behavior of dynamical systems has been firstly recognized by Poincaré [5]. If a system has the transversely intersected homoclinic orbits, then it must be chaotic. If it has the smoothly connected homoclinic orbits, then it cannot stand the perturbation and its perturbed system probably produces

( 2014 Zhang; licensee Springer. This is an Open Access article distributed under the terms of the Creative Commons Attribution License (http://creativecommons.org/licenses/by/2.0), which permits unrestricted use, distribution, and reproduction in any medium, provided the original work is properly cited. 
chaotic phenomenon. Therefore, it is of practical importance to investigate the existence of homoclinic orbits of (1.1) emanating from 0.

By applying critical point theory, the authors [6-22] studied the existence of periodic solutions and subharmonic solutions for difference equations or differential equations, which show that the critical point theory is an effective method to study periodic solutions of difference equations or differential equations. In this direction, several authors [23-34] used critical point theory to study the existence of homoclinic orbits for difference equations. Motivated mainly by the ideas of $[1-4,35]$, we will consider homoclinic solutions of (1.1) by the mountain pass theorem and the symmetric mountain pass theorem. More precisely, we obtain the following main results, which seem not to have been considered in the literature.

Theorem 1.1 Suppose that $a$ and $W$ satisfy the following conditions:

(A) Let $1<p<(q+2) / 2$ and $q>2, a: \mathbb{Z} \rightarrow(0,+\infty)$ is a positive function on $\mathbb{Z}$ such that for all $n \in \mathbb{Z}$

$$
a(n) \geq \alpha|n|^{\beta}, \quad \alpha>0, \beta>(q-2 p+2) / p .
$$

(W1) $W(n, x)=W_{1}(n, x)-W_{2}(n, x), W_{1}, W_{2}$ are continuously differentiable in $x$, and there is a bounded set $J \subset \mathbb{Z}$ such that

$$
\frac{1}{a(n)}|\nabla W(n, x)|=o\left(|x|^{q-p+1}\right) \quad \text { as } x \rightarrow 0
$$

uniformly in $n \in \mathbb{Z} \bigvee$.

(W2) There is a constant $\mu>q-p+2$ such that

$$
0<\mu W_{1}(n, x) \leq\left(\nabla W_{1}(n, x), x\right), \quad \forall(n, x) \in \mathbb{Z} \times \mathbb{R}^{N} \backslash\{0\}
$$

(W3) $W_{2}(n, 0)=0$ and there exists a constant $\varrho \in(q-p+2, \mu)$ such that

$$
W_{2}(n, x) \geq 0, \quad\left(\nabla W_{2}(n, x), x\right) \leq \varrho W_{2}(n, x), \quad \forall(n, x) \in \mathbb{Z} \times \mathbb{R}^{N}
$$

Then problem (1.1) has one nontrivial homoclinic solution.

Theorem 1.2 Suppose that $a$ and W satisfy (A), (W2) and the following conditions:

$(\mathrm{W} 1)^{\prime} W(n, x)=W_{1}(n, x)-W_{2}(n, x), W_{1}, W_{2}$ are continuously differentiable in $x$, and

$$
\frac{1}{a(n)}|\nabla W(n, x)|=o\left(|x|^{q-p+1}\right) \quad \text { as } x \rightarrow 0
$$

uniformly in $n \in \mathbb{Z}$.

(W3)' $W_{2}(n, 0)=0$ and there exists a constant $\varrho \in(q-p+2, \mu)$ such that

$$
\left(\nabla W_{2}(n, x), x\right) \leq \varrho W_{2}(n, x), \quad \forall(n, x) \in \mathbb{Z} \times \mathbb{R}^{N} .
$$

Then problem (1.1) has one nontrivial homoclinic solution. 
Theorem 1.3 Suppose that $a$ and W satisfy (A), (W1)-(W3) and

(W4) $W(n,-x)=W(n, x), \forall(n, x) \in \mathbb{Z} \times \mathbb{R}^{N}$.

Then problem (1.1) has an unbounded sequence of homoclinic solutions.

Theorem 1.4 Suppose that a and W satisfy (A), (W1)', (W2), (W3)' and (W4). Then problem (1.1) has an unbounded sequence of homoclinic solutions.

The rest of this paper is organized as follows: in Section 2, some preliminaries are presented and we establish an embedding result. In Section 3, we give the proofs of our results. In Section 4, some examples are given to illustrate our results.

\section{Preliminaries}

Let

$$
\begin{aligned}
& S=\left\{\{u(n)\}_{n \in \mathbb{Z}}: u(n) \in \mathbb{R}^{N}, n \in \mathbb{Z}\right\}, \\
& W=\left\{u \in S: \sum_{n \in \mathbb{Z}}\left[|\Delta u(n-1)|^{p}+|u(n)|^{p}\right]<+\infty\right\},
\end{aligned}
$$

and for $u \in W$, let

$$
\|u\|=\left\{\sum_{n \in \mathbb{Z}}\left[|\Delta u(n-1)|^{p}+|u(n)|^{p}\right]\right\}^{1 / p}
$$

Then $W$ is a uniform convex Banach space with this norm. As usual, for $1 \leq p<+\infty$, let

$$
l^{p}\left(\mathbb{Z}, \mathbb{R}^{N}\right)=\left\{u \in S: \sum_{n \in \mathbb{Z}}|u(n)|^{p}<+\infty\right\}, \quad l^{\infty}\left(\mathbb{Z}, \mathbb{R}^{N}\right)=\left\{u \in S: \sup _{n \in \mathbb{Z}}|u(n)|<+\infty\right\}
$$

and their norms are given by

$$
\begin{aligned}
& \|u\|_{l^{p}}=\left(\sum_{n \in \mathbb{Z}}|u(n)|^{p}\right)^{1 / p}, \quad \forall u \in l^{p}\left(\mathbb{Z}, \mathbb{R}^{N}\right), \\
& \|u\|_{\infty}=\sup \{|u(n)|: n \in \mathbb{Z}\}, \quad \forall u \in l^{\infty}\left(\mathbb{Z}, \mathbb{R}^{N}\right),
\end{aligned}
$$

respectively.

If $\sigma$ is a positive function on $\mathbb{Z}$ and $1<s<+\infty$, let

$$
l_{\sigma}^{s}=l_{\sigma}^{s}\left(\mathbb{Z}, \mathbb{R}^{N} ; \sigma\right)=\left\{\left.u \in l_{\mathrm{loc}}^{1}\left(\mathbb{Z}, \mathbb{R}^{N}\right)\left|\sum_{n \in Z} \sigma(n)\right| u(n)\right|^{s}<+\infty\right\} .
$$

$l_{\sigma}^{s}$ equipped with the norm

$$
\|u\|_{s, \sigma}=\left(\sum_{n \in Z} \sigma(n)|u(n)|^{s}\right)^{1 / s}
$$

is a reflexive Banach space. 
Set $E=W \cap l_{a}^{q-p+2}$, where $a$ is the function given in condition (A). Then $E$ with its standard norm $\|\cdot\|$ is a reflexive Banach space. The functional $\varphi$ corresponding to (1.1) on $E$ is given by

$$
\varphi(u)=\sum_{n \in \mathbb{Z}}\left[\frac{1}{p}|\Delta u(n-1)|^{p}+\frac{a(n)}{q-p+2}|u(n)|^{q-p+2}-W(n, u(n))\right], \quad u \in E .
$$

Clearly, it follows from (W1) or (W1)' that $\varphi: E \rightarrow \mathbb{R}$. By Theorem 2.1 of [36], we can deduce that the map

$$
u \rightarrow a(n)|u(n)|^{q-p} u(n)
$$

is continuous from $l_{a}^{q-p+2}$ in the dual space $l_{a^{-1 /(q-p+1)}}^{p_{1}}$, where $p_{1}=(q-p+2) /(q-p+1)$. As the embeddings $E \subset W \subset l^{\gamma}$ for all $\gamma \geq p$ are continuous, if (A) and (W1) or (W1)' hold, then $\varphi \in C^{1}(E, \mathbb{R})$ and one can easily check that

$$
\begin{aligned}
\left\langle\varphi^{\prime}(u), v\right\rangle= & \sum_{n \in \mathbb{Z}}\left[|\Delta u(n-1)|^{p-2}(\Delta u(n-1), \Delta v(n-1))+a(n)|u(n)|^{q-p}(u(n), v(n))\right] \\
& -\sum_{n \in \mathbb{Z}}(\nabla W(n, u(n)), v(n)), \quad u \in E .
\end{aligned}
$$

Furthermore, the critical points of $\varphi$ in $E$ are classical solutions of (1.1) with $u( \pm \infty)=0$.

Lemma 2.1 [23] For $u \in E$

$$
\|u\|_{\infty} \leq\|u\|_{l^{p}} \leq 2\|u\|
$$

Lemma 2.2 If a satisfies assumption (A), then

the embedding $l_{a}^{q-p+2} \subset l^{p}$ is continuous.

Moreover, there exists a Sobolev space Z such that

the embeddings $l_{a}^{q-p+2} \subset Z \subset l^{p}$ are continuous,

the embedding $W \cap Z \subset l^{p}$ is compact.

Proof Let $\theta=(q-p+2) /(q-2 p+2), \theta^{\prime}=(q-p+2) / p$, we have

$$
\begin{aligned}
\|u\|_{l^{p}}^{p} & =\sum_{n \in \mathbb{Z}}[a(n)]^{-1 / \theta^{\prime}}[a(n)]^{1 / \theta^{\prime}}|u(n)|^{p} \\
& \leq\left(\sum_{n \in \mathbb{Z}}[a(n)]^{-\theta / \theta^{\prime}}\right)^{1 / \theta}\left(\sum_{n \in \mathbb{Z}} a(n)|u(n)|^{p \theta^{\prime}}\right)^{1 / \theta^{\prime}} \\
& =a_{1}\left(\sum_{n \in \mathbb{Z}} a(n)|u(n)|^{q-p+2}\right)^{p / q-p+2} \\
& =a_{1}\|u\|_{q-p+2, a}^{p}
\end{aligned}
$$

where $a_{1}=\left(\sum_{n \in \mathbb{Z}}[a(n)]^{-p /(q-2 p+2)}\right)^{(q-2 p+2) /(q-p+2)}<+\infty$ from (A). Then (2.4) holds. 
By (A), there exists a positive function $\rho$ such that $\rho(n) \rightarrow+\infty$ as $|n| \rightarrow+\infty$ and

$$
a_{2}=\left(\sum_{n \in \mathbb{Z}}[\rho(n)]^{\theta}[a(n)]^{-\theta / \theta^{\prime}}\right)^{1 / \theta}<+\infty .
$$

Since

$$
\begin{aligned}
\|u\|_{p, \rho}^{p} & =\sum_{n \in \mathbb{Z}} \rho(n)|u(n)|^{p}=\sum_{n \in \mathbb{Z}} \rho(n)[a(n)]^{-1 / \theta^{\prime}}[a(n)]^{1 / \theta^{\prime}}|u(n)|^{p} \\
& \leq\left(\sum_{n \in \mathbb{Z}}[\rho(n)]^{\theta}[a(n)]^{-\theta / \theta^{\prime}}\right)^{1 / \theta}\left(\sum_{n \in \mathbb{Z}} a(n)|u(n)|^{q-p+2}\right)^{1 / \theta^{\prime}} \\
& =a_{2}\|u\|_{q-p+2, a}^{p}
\end{aligned}
$$

(2.5) holds by taking $Z=l_{\rho}^{p}$.

Finally, as $W \cap Z$ is the weighted Sobolev space $\Gamma^{1, p}(\mathbb{Z}, \rho, 1)$, it follows from [36] that (2.6) holds.

The following two lemmas are the mountain pass theorem and the symmetric mountain pass theorem, which are useful in the proofs of our theorems.

Lemma 2.3 [37] Let $E$ be a real Banach space and $I \in C^{1}(E, \mathbb{R})$ satisfying the (PS)condition. Suppose $I(0)=0$ and

(i) There exist constants $\rho, \alpha>0$ such that $I_{\partial B_{\rho}(0)} \geq \alpha$.

(ii) There exists an $e \in E \backslash \bar{B}_{\rho}(0)$ such that $I(e) \leq 0$.

Then I possesses a critical value $c \geq \alpha$ which can be characterized as

$$
c=\inf _{h \in \Phi} \max _{s \in[0,1]} I(h(s))
$$

where $\Phi=\{h \in C([0,1], E) \mid h(0)=0, h(1)=e\}$, and $B_{\rho}(0)$ is an open ball in $E$ of radius $\rho$ centered at 0 .

Lemma 2.4 [37] Let E be a real Banach space and $I \in C^{1}(E, \mathbb{R})$ with I even. Assume that $I(0)=0$ and I satisfies (PS)-condition, (i) of Lemma 2.3 and the following condition:

(iii) For each finite dimensional subspace $E^{\prime} \subset E$, there is $r=r\left(E^{\prime}\right)>0$ such that $I(u) \leq 0$ for $u \in E^{\prime} \backslash B_{r}(0), B_{r}(0)$ is an open ball in $E$ of radius $r$ centered at 0 .

Then I possesses an unbounded sequence of critical values.

Lemma 2.5 Assume that (W2) and (W3) or (W3)' hold. Then for every $(n, x) \in \mathbb{Z} \times \mathbb{R}^{N}$,

(i) $s^{-\mu} W_{1}(n, s x)$ is nondecreasing on $(0,+\infty)$;

(ii) $s^{-\varrho} W_{2}(n, s x)$ is nonincreasing on $(0,+\infty)$.

The proof of Lemma 2.5 is routine and we omit it. In the following, $C_{i}(i=1,2, \ldots)$ denote different positive constants.

\section{Proofs of theorems}

Proof of Theorem 1.1 Firstly, we prove that the functional $\varphi$ satisfies the (PS)-condition. Let $\left\{u_{k}\right\} \subset E$ satisfying $\varphi\left(u_{k}\right)$ is bounded and $\varphi^{\prime}\left(u_{k}\right) \rightarrow 0$ as $k \rightarrow \infty$. Hence, there exists a 
constant $C_{1}>0$ such that

$$
\left|\varphi\left(u_{k}\right)\right| \leq C_{1}, \quad\left\|\varphi^{\prime}\left(u_{k}\right)\right\|_{E^{*}} \leq \mu C_{1} .
$$

From (2.1), (2.2), (3.1), (W2), and (W3), we have

$$
\begin{aligned}
p C_{1}+p C_{1}\left\|u_{k}\right\| \geq & p \varphi\left(u_{k}\right)-\frac{p}{\mu}\left\langle\varphi^{\prime}\left(u_{k}\right), u_{k}\right\rangle \\
= & \frac{\mu-p}{\mu}\left\|\Delta u_{k}\right\|_{l^{p}}^{p}+p \sum_{n \in \mathbb{Z}}\left[W_{2}\left(n, u_{k}(n)\right)-\frac{1}{\mu}\left(\nabla W_{2}\left(n, u_{k}(n)\right), u_{k}(n)\right)\right] \\
& -p \sum_{n \in \mathbb{Z}}\left[W_{1}\left(n, u_{k}(n)\right)-\frac{1}{\mu}\left(\nabla W_{1}\left(n, u_{k}(n)\right), u_{k}(n)\right)\right] \\
& +\left(\frac{p}{q-p+2}-\frac{p}{\mu}\right) \sum_{n \in \mathbb{Z}} a(n)\left|u_{k}(n)\right|^{q-p+2} \\
\geq & \frac{\mu-p}{\mu}\left\|\Delta u_{k}\right\|_{l^{p}}^{p}+\left(\frac{p}{q-p+2}-\frac{p}{\mu}\right)\left\|u_{k}\right\|_{q-p+2, a}^{q-p+2} .
\end{aligned}
$$

It follows from Lemma $2.2, p<(q+2) / 2, \mu>q-p+2$, and (3.2) that there exists a constant $C_{2}>0$ such that

$$
\left\|u_{k}\right\| \leq C_{2}, \quad k \in \mathbb{N} .
$$

Now we prove that $u_{k} \rightarrow u$ in $E$. Passing to a subsequence if necessary, it can be assumed that $u_{k} \rightarrow u$ in $E$. For any given $\varepsilon>0$, by (W1), we can choose $\delta \in(0,1)$ such that

$$
|\nabla W(n, x)| \leq \varepsilon a(n)|x|^{q-p+1} \quad \text { for } n \in \mathbb{Z} \bigvee \text { and }|x| \leq \delta .
$$

Since $u \in E$, we can also choose a positive integer $K>\max \{|k|: k \in J\}$ such that

$$
|u(n)| \leq \delta \quad \text { for }|n| \geq K .
$$

Hence,

$$
|\nabla W(n, u(n))| \leq \varepsilon a(n)|u(n)|^{q-p+1} \quad \text { for } n \in \mathbb{Z} \bigvee \text { and }|u(n)| \leq \delta \text {. }
$$

Furthermore,

$$
\left|\nabla W\left(n, u_{k}(n)\right)\right| \leq \varepsilon a(n)\left|u_{k}(n)\right|^{q-p+1} \quad \text { for } n \in \mathbb{Z} \backslash \text { and }\left|u_{k}(n)\right| \leq \delta \text {. }
$$

Hence, from (3.5) and (3.6), we have

$$
\begin{aligned}
& \left|\nabla W\left(n, u_{k}(n)\right)-\nabla W(n, u(n))\right|^{p^{\prime}} \\
& \quad \leq\left[\varepsilon a(n)\left(\left|u_{k}(n)\right|^{q-p+1}+|u(n)|^{q-p+1}\right)\right]^{p^{\prime}} \\
& \quad \leq\left[\varepsilon 2^{q-p+1} a(n)\left|u_{k}(n)-u(n)\right|^{q-p+1}+\varepsilon\left(1+2^{q-p+1}\right) a(n)|u(n)|^{q-p+1}\right]^{p^{\prime}}
\end{aligned}
$$




$$
\begin{aligned}
\leq & 2^{p^{\prime}(q-p+2)} \varepsilon^{p^{\prime}}[a(n)]^{p^{\prime}}\left|u_{k}(n)-u(n)\right|^{p^{\prime}(q-p+1)} \\
& +2^{p^{\prime}} \varepsilon^{p^{\prime}}\left(1+2^{q-p+1}\right)^{p^{\prime}}[a(n)]^{p^{\prime}}|u(n)|^{p^{\prime}(q-p+1)} \\
:= & g_{k}(n),
\end{aligned}
$$

where $p^{\prime}=p /(p-1)$. Moreover, since $a(n)$ is a positive function on $\mathbb{Z}, p<q-p+2$, and $u_{k}(n) \rightarrow u(n)$ for almost every $n \in \mathbb{Z}$, we have

$$
\lim _{k \rightarrow \infty} g_{k}(n)=2^{p^{\prime}} \varepsilon^{p^{\prime}}\left(1+2^{q-p+1}\right)^{p^{\prime}}[a(n)]^{p^{\prime}}|u(n)|^{p(q-p+1)}:=g(n), \quad \text { for a.e. } n \in \mathbb{Z},
$$

and

$$
\begin{aligned}
\lim _{k \rightarrow \infty} \sum_{n \in \mathbb{Z}} g_{k}(n)= & \lim _{k \rightarrow \infty} \sum_{n \in \mathbb{Z}}\left[2^{p^{\prime}(q-p+2)} \varepsilon^{p^{\prime}}[a(n)]^{p^{\prime}}\left|u_{k}(n)-u(n)\right|^{p^{\prime}(q-p+1)}\right. \\
& \left.+2^{p^{\prime}} \varepsilon^{p^{\prime}}\left(1+2^{q-p+1}\right)^{p^{\prime}}[a(n)]^{p^{\prime}}|u(n)|^{p^{\prime}(q-p+1)}\right] \\
= & 2^{p^{\prime}(q-p+2)} \varepsilon^{p^{\prime}} \lim _{k \rightarrow \infty} \sum_{n \in \mathbb{Z}}[a(n)]^{p^{\prime}}\left|u_{k}(n)-u(n)\right|^{p^{\prime}(q-p+1)} \\
& +2^{p^{\prime}} \varepsilon^{p^{\prime}}\left(1+2^{q-p+1}\right)^{p^{\prime}} \sum_{n \in \mathbb{Z}}[a(n)]^{p^{\prime}}|u(n)|^{p^{\prime}(q-p+1)} \\
= & 2^{p^{\prime}} \varepsilon^{p^{\prime}}\left(1+2^{q-p+1}\right)^{p^{\prime}} \sum_{n \in \mathbb{Z}}[a(n)]^{p^{\prime}}|u(n)|^{p^{\prime}(q-p+1)} \\
= & \sum_{n \in \mathbb{Z}} g(n)<+\infty .
\end{aligned}
$$

It follows from (3.7), (3.8), (3.9), and the Lebesgue dominated convergence theorem that

$$
\lim _{k \rightarrow \infty} \sum_{n \in \mathbb{Z}}\left|\nabla W\left(n, u_{k}(n)\right)-\nabla W(n, u(n))\right|^{p^{\prime}}=0
$$

This shows that

$$
\nabla W\left(n, u_{k}\right) \rightarrow \nabla W(n, u) \quad \text { in } l^{p^{\prime}}\left(\mathbb{Z}, \mathbb{R}^{N}\right)
$$

From (2.2), we have

$$
\begin{aligned}
& \left.\left\langle\varphi^{\prime}\left(u_{k}\right)-\varphi^{\prime}(u), u_{k}-u\right)\right\rangle \\
& =\sum_{n \in \mathbb{Z}}\left(\left|\Delta u_{k}(n-1)\right|^{p-2} \Delta u_{k}(n-1)-|\Delta u(n-1)|^{p-2} \Delta u(n-1),\right. \\
& \left.\quad \Delta u_{k}(n-1)-\Delta u(n-1)\right) \\
& \quad+\sum_{n \in \mathbb{Z}} a(n)\left(\left|u_{k}(n)\right|^{q-p} u_{k}(n)-|u(n)|^{q-p} u(n)\right)\left(u_{k}(n)-u(n)\right) \\
& \quad-\sum_{n \in \mathbb{Z}}\left(\nabla W\left(n, u_{k}(n)\right)-\nabla W(n, u(n)), u_{k}(n)-u(n)\right) \\
& \geq\left\|\Delta u_{k}\right\|_{l^{p}}^{p}+\|\Delta u\|_{l^{p}}^{p}-\|\Delta u\|_{p^{p}}\left\|\Delta u_{k}\right\|_{l^{p}}^{p-1}-\left\|\Delta u_{k}\right\|_{l^{p}}\|\Delta u\|_{l^{p}}^{p-1}
\end{aligned}
$$




$$
\begin{aligned}
& +\sum_{n \in \mathbb{Z}} a(n)\left(\left|u_{k}(n)\right|^{q-p} u_{k}(n)-|u(n)|^{q-p} u(n)\right)\left(u_{k}(n)-u(n)\right) \\
& -\sum_{n \in \mathbb{Z}}\left(\nabla W\left(n, u_{k}(n)\right)-\nabla W(n, u(n)), u_{k}(n)-u(n)\right) \\
= & \left(\left\|\Delta u_{k}\right\|_{l^{p}}^{p-1}-\|\Delta u\|_{l^{p}}^{p-1}\right)\left(\left\|\Delta u_{k}\right\|_{l^{p}}-\|\Delta u\|_{l^{p}}\right) \\
& +\sum_{n \in \mathbb{Z}} a(n)\left(\left|u_{k}(n)\right|^{q-p} u_{k}(n)-|u(n)|^{q-p} u(n)\right)\left(u_{k}(n)-u(n)\right) \\
& -\sum_{n \in \mathbb{Z}}\left(\nabla W\left(n, u_{k}(n)\right)-\nabla W(n, u(n)), u_{k}(n)-u(n)\right) .
\end{aligned}
$$

It is easy to see that for any $\alpha>1$ there exists a constant $C_{3}>0$ such that

$$
\left(|x|^{\alpha-1} x-|y|^{\alpha-1} y\right)(x-y) \geq C_{3}|x-y|^{\alpha+1}, \quad \forall x, y \in \mathbb{R} .
$$

Hence, we have

$$
\left(\left\|\Delta u_{k}\right\|_{l^{p}}^{p-1}-\|\Delta u\|_{l^{p}}^{p-1}\right)\left(\left\|\Delta u_{k}\right\|_{l^{p}}-\|\Delta u\|_{l^{p}}\right) \geq C_{4}\left|\left\|\Delta u_{k}\right\|_{l^{p}}-\|\Delta u\|_{l^{p}}\right|^{p}
$$

and

$$
\begin{aligned}
& \sum_{n \in \mathbb{Z}} a(n)\left(\left|u_{k}(n)\right|^{q-p} u_{k}(n)-|u(n)|^{q-p} u(n)\right)\left(u_{k}(n)-u(n)\right) \\
& \quad \geq C_{5} \sum_{n \in \mathbb{Z}} a(n)\left|u_{k}(n)-u(n)\right|^{q-p+2} .
\end{aligned}
$$

Since $\varphi^{\prime}\left(u_{k}\right) \rightarrow 0$ as $k \rightarrow+\infty, u_{k} \rightarrow u$ in $E$ and the embeddings $E \subset W \subset l^{\gamma}$ for all $\gamma \geq p$ are continuous, it follows from Lemma 2.2, (3.10), (3.11), (3.13), and (3.14) that

$$
\left\|\Delta u_{k}\right\|_{l^{p}} \rightarrow\|\Delta u\|_{l^{p}} \quad \text { as } k \rightarrow \infty
$$

and

$$
\sum_{n \in \mathbb{Z}} a(n)\left|u_{k}(n)\right|^{q-p+2} \rightarrow \sum_{n \in \mathbb{Z}} a(n)|u(n)|^{q-p+2} \quad \text { as } k \rightarrow \infty .
$$

Hence, we have $u_{k} \rightarrow u$ in $E$ by (3.15) and (3.16). This shows that $\varphi$ satisfies the (PS)condition.

Secondly, we prove that there exist $\rho, \alpha>0$ such that $\varphi_{\partial B_{\rho}(0)} \geq \alpha$. From (W1), there exists $\delta_{1} \in(0,1)$ such that

$$
|\nabla W(n, x)| \leq \frac{1}{p} a(n)|x|^{q-p+1} \quad \text { for }|n| \geq \mathbb{Z} \bigvee \text { and }|x| \leq \delta_{1} .
$$

From (3.17), we have

$$
|W(n, x)| \leq \frac{1}{p(q-p+2)} a(n)|x|^{q-p+2} \quad \text { for }|n| \geq \mathbb{Z} \backslash \text { and }|x| \leq \delta_{1}
$$


Let

$$
C_{6}=\sup \left\{\frac{W_{1}(n, x)}{a(n)}\left|n \in J, x \in \mathbb{R}^{N},\right| x \mid=1\right\} .
$$

Set $\sigma=\min \left\{1 /\left(p(q-p+2) C_{6}+1\right)^{1 /(\mu-q+p-2)}, \delta_{1}\right\}$ and $\|u\|=\sigma / 2:=\rho$, it follows from (2.3) that

$$
\|u\|_{\infty} \leq 2\|u\| \leq \sigma,
$$

which shows that $|u(n)| \leq \sigma \leq \delta_{1}<1$. From Lemma 2.5(i) and (3.19), we have

$$
\begin{aligned}
\sum_{n \in J} W_{1}(n, u(n)) & \leq \sum_{\{n \in J: u(n) \neq 0\}} W_{1}\left(n, \frac{u(n)}{|u(n)|}\right)|u(n)|^{\mu} \\
& \leq C_{6} \sum_{n \in J} a(n)|u(n)|^{\mu} \leq C_{6} \sigma^{\mu-q+p-2} \sum_{n \in J} a(n)|u(n)|^{q-p+2} \\
& \leq \frac{1}{p(q-p+2)} \sum_{n \in J} a(n)|u(n)|^{q-p+2} .
\end{aligned}
$$

It follows from (W3), (3.18), and (3.20) that

$$
\begin{aligned}
\varphi(u)= & \frac{1}{p} \sum_{n \in \mathbb{Z}}|\Delta u(n-1)|^{p}+\sum_{n \in \mathbb{Z}} \frac{a(n)}{q-p+2}|u(n)|^{q-p+2}-\sum_{n \in \mathbb{Z}} W(n, u(n)) \\
= & \frac{1}{p}\|\Delta u\|_{l^{p}}^{p}+\frac{1}{q-p+2}\|u\|_{q-p+2, a}^{q-p+2}-\sum_{\mathbb{Z} \backslash} W(n, u(n))-\sum_{n \in J} W(n, u(n)) \\
\geq & \frac{1}{p}\|\Delta u\|_{l^{p}}^{p}+\frac{1}{q-p+2}\|u\|_{q-p+2, a}^{q-p+2}-\sum_{n \in J} W_{1}(n, u(n)) \\
& -\sum_{\mathbb{Z} \backslash} \frac{1}{p(q-p+2)} a(n)|u(n)|^{q-p+2} \\
\geq & \frac{1}{p}\|\Delta u\|_{l^{p}}^{l^{p}}+\frac{1}{q-p+2}\|u\|_{q-p+2, a}^{q-p+2}-\frac{1}{p(q-p+2)} \sum_{n \in J} a(n)|u(n)|^{q-p+2} \\
& -\sum_{\mathbb{Z} \backslash} \frac{1}{p(q-p+2)} a(n)|u(n)|^{q-p+2} \\
= & \frac{1}{p}\|\Delta u\|_{l^{p}}^{p}+\frac{p-1}{p(q-p+2)}\|u\|_{q-p+2, a}^{q-p+2} .
\end{aligned}
$$

Therefore, we can choose a constant $\alpha>0$ depending on $\rho$ such that $\varphi(u) \geq \alpha$ for any $u \in E$ with $\|u\|=\rho$.

Thirdly, we prove that assumption (ii) of Lemma 2.3 holds. From Lemma 2.5(ii) and (2.3), we have for any $u \in E$

$$
\begin{aligned}
\sum_{n \in[-3,3]} W_{2}(n, u(n)) & =\sum_{\{n \in[-3,3]:|u(n)|>1\}} W_{2}(n, u(n))+\sum_{\{n \in[-3,3]:|u(n)| \leq 1\}} W_{2}(n, u(n)) \\
& \leq \sum_{\{n \in[-3,3]:|u(n)|>1\}} W_{2}\left(n, \frac{u(n)}{|u(n)|}\right)|u(n)|^{\varrho}+\sum_{n \in[-3,3]} \max _{|x| \leq 1} W_{2}(n, x)
\end{aligned}
$$




$$
\begin{aligned}
& \leq\|u\|_{\infty}^{\varrho} \sum_{n \in[-3,3]} \max _{|x|=1} W_{2}(n, x)+\sum_{n \in[-3,3]} \max _{|x| \leq 1} W_{2}(n, x) \\
& \leq 2^{\varrho}\|u\|^{\varrho} \sum_{n \in[-3,3]} \max _{|x|=1} W_{2}(n, x)+\sum_{n \in[-3,3]} \max _{|x| \leq 1} W_{2}(n, x) \\
& =C_{7}\|u\|^{\varrho}+C_{8},
\end{aligned}
$$

where $C_{7}=2^{\varrho} \sum_{n \in[-3,3]} \max _{|x|=1} W_{2}(n, x), C_{8}=\sum_{n \in[-3,3]} \max _{|x| \leq 1} W_{2}(n, x)$. Take $\omega \in E$ such that

$$
|\omega(n)|= \begin{cases}1, & \text { for }|n| \leq 1 \\ 0, & \text { for }|n| \geq 3\end{cases}
$$

and $|\omega(n)| \leq 1$ for $|n| \in(1,3]$. For $s>1$, from Lemma 2.5(i) and (3.22), we get

$$
\sum_{n \in[-1,1]} W_{1}(n, s \omega(n)) \geq s^{\mu} \sum_{n \in[-1,1]} W_{1}(n, \omega(n))=C_{9} s^{\mu}
$$

where $C_{9}=\sum_{n \in[-1,1]} W_{1}(n, \omega(n))>0$. From (W3), (2.1), (3.21), (3.22), (3.23), we have for $s>1$

$$
\begin{aligned}
\varphi(s \omega) & =\frac{s^{p}}{p}\|\Delta \omega\|_{l^{p}}^{p}+\frac{s^{q-p+2}}{q-p+2}\|\omega\|_{q-p+2, a}^{q-p+2}+\sum_{n \in \mathbb{Z}}\left[W_{2}(n, s \omega(n))-W_{1}(n, s \omega(n))\right] \\
& \leq \frac{s^{p}}{p}\|\Delta \omega\|_{l^{p}}^{p}+\frac{s^{q-p+2}}{q-p+2}\|\omega\|_{q-p+2, a}^{q-p+2}+\sum_{n \in[-3,3]} W_{2}(n, s \omega(n))-\sum_{n \in[-1,1]} W_{1}(n, s \omega(n)) \\
& \leq \frac{s^{p}}{p}\|\Delta \omega\|_{l^{p}}^{p}+\frac{s^{q-p+2}}{q-p+2}\|\omega\|_{q-p+2, a}^{q-p+2}+C_{7} s^{\varrho}\|\omega\|^{\varrho}+C_{8}-C_{9} s^{\mu} .
\end{aligned}
$$

Since $\mu>\varrho>q-p+2$ and $C_{9}>0$, it follows from (3.24) that there exists $s_{1}>1$ such that $\left\|s_{1} \omega\right\|>\rho$ and $\varphi\left(s_{1} \omega\right)<0$. Let $e=s_{1} \omega(n)$, then $e \in E,\|e\|=\left\|s_{1} \omega\right\|>\rho$, and $\varphi(e)=\varphi\left(s_{1} \omega\right)<0$.

By Lemma 2.3, $\varphi$ has a critical value $c>\alpha$ given by

$$
c=\inf _{g \in \Phi} \max _{s \in[0,1]} \varphi(g(s))
$$

where

$$
\Phi=\{g \in C([0,1], E): g(0)=0, g(1)=e\}
$$

Hence, there exists $u^{*} \in E$ such that

$$
\varphi\left(u^{*}\right)=c, \quad \varphi^{\prime}\left(u^{*}\right)=0 .
$$

The function $u^{*}$ is a desired solution of problem (1.1). Since $c>0, u^{*}$ is a nontrivial homoclinic solution. The proof is complete.

Proof of Theorem 1.2 In the proof of Theorem 1.1, the condition $W_{2}(t, x) \geq 0$ in (W3) is only used in the proofs of (3.3) and assumption (i) of Lemma 2.3. Therefore, we only need 
to prove that (3.3) and assumption (i) of Lemma 2.3 still hold if we use (W1)' and (W3)' instead of (W1) and (W3), respectively. We first prove that (3.3) holds. From (W2), (W3)', (2.1), (2.2), and (3.1), we have

$$
\begin{aligned}
p(q-p+2) C_{1}+\frac{p(q-p+2) C_{1} \mu}{\varrho}\left\|u_{k}\right\| \\
\geq p(q-p+2) \varphi\left(u_{k}\right)-\frac{p(q-p+2)}{\varrho}\left\langle\varphi^{\prime}\left(u_{k}\right), u_{k}\right\rangle \\
=\frac{(\varrho-p)(q-p+2)}{\varrho}\left\|\Delta u_{k}\right\|_{l^{p}}^{p} \\
\quad+p(q-p+2) \sum_{n \in \mathbb{Z}}\left[W_{2}\left(n, u_{k}(n)\right)-\frac{1}{\varrho}\left(\nabla W_{2}\left(n, u_{k}(n)\right), u_{k}(n)\right)\right] \\
\quad-p(q-p+2) \sum_{n \in \mathbb{Z}}\left[W_{1}\left(n, u_{k}(n)\right)-\frac{1}{\varrho}\left(\nabla W_{1}\left(n, u_{k}(n)\right), u_{k}(n)\right)\right] \\
\quad+p\left(1-\frac{q-p+2}{\varrho}\right) \sum_{n \in \mathbb{Z}} a(n)\left|u_{k}(n)\right|^{q-p+2} \\
\geq \\
\quad \frac{(\varrho-p)(q-p+2)}{\varrho}\left\|\Delta u_{k}\right\|_{l^{p}}^{p}+p\left(1-\frac{q-p+2}{\varrho}\right)\left\|u_{n}\right\|_{q-p+2, a}^{q-p+2},
\end{aligned}
$$

which implies that there exists a constant $C_{2}>0$ such that (3.3) holds. Next, we prove that assumption (i) of Lemma 2.3 still holds. From (W1)', there exists $\delta_{2} \in(0,1)$ such that

$$
|\nabla W(n, x)| \leq \frac{1}{p} a(n)|x|^{q-p+1} \quad \text { for } n \in \mathbb{Z} \text { and }|x| \leq \delta_{2} .
$$

By (3.26), we have

$$
|W(n, x)| \leq \frac{1}{p(q-p+2)} a(n)|x|^{q-p+2} \quad \text { for } n \in \mathbb{Z} \text { and }|x| \leq \delta_{2} .
$$

Let $0<\sigma \leq \delta_{2}$ and $\|u\|=\sigma / 2:=\rho$, it follows from (2.3) that

$$
\|u\|_{\infty} \leq 2\|u\| \leq \sigma
$$

which shows that $|u(n)| \leq \sigma \leq \delta_{2}<1$. It follows from (2.1) and (3.27) that

$$
\begin{aligned}
\varphi(u) & =\frac{1}{p} \sum_{n \in \mathbb{Z}}|\Delta u(n-1)|^{p}+\sum_{n \in \mathbb{Z}} \frac{a(n)}{q-p+2}|u(n)|^{q-p+2}-\sum_{n \in \mathbb{Z}} W(n, u(n)) \\
& \geq \frac{1}{p}\|\Delta u\|_{l^{p}}^{p}+\frac{1}{q-p+2}\|u\|_{q-p+2, a}^{q-p+2}-\sum_{n \in \mathbb{Z}} \frac{1}{p(q-p+2)} a(n)|u(n)|^{q-p+2} \\
& =\frac{1}{p}\|\Delta u\|_{l^{p}}^{p}+\frac{p-1}{p(q-p+2)}\|u\|_{q-p+2, a}^{q-p+2} .
\end{aligned}
$$

Therefore, we can choose a constant $\alpha>0$ depending on $\rho$ such that $\varphi(u) \geq \alpha$ for any $u \in E$ with $\|u\|=\rho$. The proof of Theorem 1.2 is complete. 
Proof of Theorem 1.3 Condition (W4) shows that $\varphi$ is even. In view of the proof of Theorem 1.1, we know that $\varphi \in C^{1}(E, \mathbb{R})$ and satisfies (PS)-condition and assumption (i) of Lemma 2.3. Now, we prove that assumption (iii) of Lemma 2.4 holds. Let $E^{\prime}$ be a finite dimensional subspace of $E$. Since all norms of a finite dimensional space are equivalent, there exists $C_{10}>0$ such that

$$
\|u\| \leq C_{10}\|u\|_{\infty}
$$

Assume that $\operatorname{dim} E^{\prime}=m$ and $\left\{u_{1}, u_{2}, \ldots, u_{m}\right\}$ is a base of $E^{\prime}$ such that

$$
\left\|u_{i}\right\|=C_{10}, \quad i=1,2, \ldots, m
$$

For any $u \in E^{\prime}$, there exists $\lambda_{i} \in \mathbb{R}, i=1,2, \ldots, m$ such that

$$
u(n)=\sum_{i=1}^{m} \lambda_{i} u_{i}(n) \quad \text { for } n \in \mathbb{Z}
$$

Let

$$
\|u\|_{*}=\sum_{i=1}^{m}\left|\lambda_{i}\right|\left\|u_{i}\right\|
$$

It is easy to see that $\|\cdot\|_{*}$ is a norm of $E^{\prime}$. Hence, there exists a constant $C_{11}>0$ such that $C_{11}\|u\|_{*} \leq\|u\|$. Since $u_{i} \in E$, by Lemma 2.2 , we can choose $K_{1}>K$ such that

$$
\left|u_{i}(n)\right|<\frac{C_{11} \delta_{1}}{1+C_{11}}, \quad|n|>K_{1}, i=1,2, \ldots, m,
$$

where $\delta_{1}$ is given in (3.17). Let

$$
\Theta=\left\{\sum_{i=1}^{m} \lambda_{i} u_{i}(n): \lambda_{i} \in \mathbb{R}, i=1,2, \ldots, m ; \sum_{i=1}^{m}\left|\lambda_{i}\right|=1\right\}=\left\{u \in E^{\prime}:\|u\|_{*}=C_{10}\right\} .
$$

Hence, for $u \in \Theta$, let $n_{0}=n_{0}(u) \in \mathbb{Z}$ such that

$$
\left|u\left(n_{0}\right)\right|=\|u\|_{\infty} .
$$

It follows from (3.28)-(3.31), (3.33), and (3.34) that

$$
\begin{aligned}
C_{10} C_{11} & =C_{10} C_{11} \sum_{i=1}^{m}\left|\lambda_{i}\right|=C_{11} \sum_{i=1}^{m}\left|\lambda_{i}\right|\left\|u_{i}\right\|=C_{11}\|u\|_{*} \\
& \leq\|u\| \leq C_{10}\|u\|_{\infty}=C_{10}\left|u\left(n_{0}\right)\right| \\
& \leq C_{10} \sum_{i=1}^{m}\left|\lambda_{i}\right|\left|u_{i}\left(n_{0}\right)\right|, \quad u \in \Theta .
\end{aligned}
$$

This shows that $\left|u\left(n_{0}\right)\right| \geq C_{11}$ and there exists $i_{0} \in\{1,2, \ldots, m\}$ such that $\left|u_{i_{0}}\left(n_{0}\right)\right| \geq C_{11}$, which together with (3.32), implies that $\left|n_{0}\right| \leq K_{1}$. Let

$$
\gamma=\min \left\{W_{1}(n, x):-K_{1} \leq n \leq K_{1},|x| \leq C_{11}\right\} .
$$


Since $W_{1}(n, x)>0$ for all $n \in \mathbb{Z}$ and $x \in \mathbb{R}^{N} \backslash\{0\}$, and $W_{1}(n, x)$ is continuous in $x$, it follows that $\gamma>0$. For any $u \in E$, from Lemma 2.5(ii) and (2.3), we have

$$
\begin{aligned}
\sum_{n=-K_{1}}^{K_{1}} W_{2}(n, u(n)) & =\sum_{\left\{n \in\left[-K_{1}, K_{1}\right]:|u(n)|>1\right\}} W_{2}(n, u(n))+\sum_{\left\{n \in\left[-K_{1}, K_{1}\right]:|u(n)| \leq 1\right\}} W_{2}(n, u(n)) \\
& \leq \sum_{\left\{n \in\left[-K_{1}, K_{1}\right]:|u(n)|>1\right\}} W_{2}\left(n, \frac{u(n)}{|u(n)|}\right)|u(n)|^{\varrho}+\sum_{n=-K_{1}}^{K_{1}} \max _{|x| \leq 1} W_{2}(n, x) \\
& \leq\|u\|_{\infty}^{\varrho} \sum_{n=-K_{1}}^{K_{1}} \max _{|x|=1} W_{2}(n, x)+\sum_{n=-K_{1}}^{K_{1}} \max _{|x| \leq 1} W_{2}(n, x) \\
& \leq 2^{\varrho}\|u\|^{\varrho} \sum_{n=-K_{1}}^{K_{1}} \max _{|x|=1} W_{2}(n, x)+\sum_{n=-K_{1}}^{K_{1}} \max _{|x| \leq 1} W_{2}(n, x) \\
& =C_{12}\|u\|^{\varrho}+C_{13},
\end{aligned}
$$

where $C_{12}=2^{\varrho} \sum_{n=-K_{1}}^{K_{1}} \max _{|x|=1} W_{2}(n, x), C_{13}=\sum_{n=-K_{1}}^{K_{1}} \max _{|x| \leq 1} W_{2}(n, x)$. It follows from Lemma 2.5(i) and (3.36) that

$$
\begin{aligned}
\sum_{n=-K_{1}}^{K_{1}} W_{1}(n, u(n)) & \geq W_{1}\left(n_{0}, u\left(n_{0}\right)\right) \\
& \geq W_{1}\left(n_{0}, \frac{C_{11} u\left(n_{0}\right)}{\left|u\left(n_{0}\right)\right|}\right)\left(\frac{\left|u\left(n_{0}\right)\right|}{C_{11}}\right)^{\mu} \\
& \geq \min _{|x| \leq 1}\left\{W_{1}\left(n_{0}, x\right)\right\} \\
& \geq \gamma \quad \text { for } u \in \Theta .
\end{aligned}
$$

By (3.18), (3.37), (3.38), and Lemma 2.5, we have for $u \in \Theta$ and $r>1$

$$
\begin{aligned}
\varphi(r u)= & \frac{r^{p}}{p}\|\Delta u\|_{p^{p}}^{p}+\frac{r^{q-p+2}}{q-p+2}\|u\|_{q-p+2, a}^{q-p+2}+\sum_{n \in \mathbb{Z}}\left[W_{2}(n, r u(n))-W_{1}(n, r u(n))\right] \\
\leq & \frac{r^{p}}{p}\|\Delta u\|_{p^{p}}^{p}+\frac{r^{q-p+2}}{q-p+2}\|u\|_{q-p+2, a}^{q-p+2}+r^{\varrho} \sum_{n \in \mathbb{Z}} W_{2}(n, u(n))-r^{\mu} \sum_{n \in \mathbb{Z}} W_{1}(n, u(n)) \\
= & \frac{r^{p}}{p}\|\Delta u\|_{p^{p}}^{p}+\frac{r^{q-p+2}}{q-p+2}\|u\|_{q-p+2, a}^{q-p+2}+r^{\varrho} \sum_{|n| \geq K_{1}} W_{2}(n, u(n)) \\
& -r^{\mu} \sum_{|n| \geq K_{1}} W_{1}(n, u(n))+r^{\varrho} \sum_{n=-K_{1}}^{K_{1}} W_{2}(n, u(n))-r^{\mu} \sum_{n=-K_{1}}^{K_{1}} W_{1}(n, u(n)) \\
\leq & \frac{r^{p}}{p}\|\Delta u\|_{p^{p}}^{p}+\frac{r^{q-p+2}}{q-p+2}\|u\|_{q-p+2, a}^{q-p+2}-r^{\varrho} \sum_{|n| \geq K_{1}} W(n, u(n)) \\
& -r^{\mu} \sum_{n=-K_{1}}^{K_{1}} W_{1}(n, u(n))+r^{\varrho} \sum_{n=-K_{1}}^{K_{1}} W_{2}(n, u(n)) \\
\leq & \frac{r^{p}}{p}\|\Delta u\|_{l^{p}}^{p}+\frac{r^{q-p+2}}{q-p+2}\|u\|_{q_{-p+2, a}}^{q-p+2}+\frac{r^{\varrho}}{p(q-p+2)} \sum_{|n| \geq K_{1}} a(n)|u(n)|^{q-p+2}
\end{aligned}
$$




$$
\begin{aligned}
& +r^{\varrho}\left(C_{12}\|u\|^{\varrho}+C_{13}\right)-\gamma r^{\mu} \\
\leq & \frac{r^{p}}{p}\|\Delta u\|_{l^{p}}^{p}+\left(\frac{r^{q-p+2}}{q-p+2}+\frac{r^{\varrho}}{p(q-p+2)}\right)\|u\|_{q-p+2, a}^{q-p+2}+r^{\varrho}\left(C_{12}\|u\|^{\varrho}+C_{13}\right)-\gamma r^{\mu} \\
\leq & \frac{r^{p}}{p} C_{10}^{p}+\left(\frac{r^{q-p+2}}{q-p+2}+\frac{r^{\varrho}}{p(q-p+2)}\right) C_{10}^{q-p+2} \\
& +C_{12}\left(r C_{10}\right)^{\varrho}+C_{13} r^{\varrho}-\gamma r^{\mu} .
\end{aligned}
$$

Since $\mu>\varrho>q-p+2>p$, we deduce that there exists $r_{0}=r_{0}\left(C_{10}, C_{11}, C_{12}, C_{13}, K, K_{1}, \varepsilon, \gamma\right)=$ $r_{0}\left(E^{\prime}\right)>1$ such that

$$
\varphi(r u)<0 \quad \text { for } u \in \Theta \text { and } r \geq r_{0} .
$$

It follows that

$$
\varphi(u)<0 \quad \text { for } u \in E^{\prime} \text { and }\|u\| \geq C_{10} r_{0},
$$

which shows that assumption (iii) of Lemma 2.4 holds. By Lemma 2.4, $\varphi$ possesses an unbounded sequence $\left\{c_{k}\right\}_{k=1}^{\infty}$ of critical values with $c_{k}=\varphi\left(u_{k}\right)$, where $u_{k}$ is such that $\varphi^{\prime}\left(u_{k}\right)=0$ for $k=1,2, \ldots$. If $\left\{\left\|u_{k}\right\|\right\}$ is bounded, then there exists $C_{14}>0$ such that

$$
\left\|u_{k}\right\| \leq C_{14} \quad \text { for } k \in \mathbb{N} .
$$

In a similar fashion to the proof of (3.5) and (3.6), for the given $\delta_{1}$ in (3.18), there exists $K_{2}>\max \{|k|: k \in J\}$ such that

$$
\left|u_{k}(n)\right| \leq \delta_{1} \quad \text { for }|n| \geq K_{2} \text { and } k \in \mathbb{N} \text {. }
$$

Hence, by (2.1), (2.3), (3.18), (3.40), and (3.41), we have

$$
\begin{aligned}
\frac{1}{p} & \left\|\Delta u_{k}\right\|_{p^{p}}^{p}+\frac{1}{q-p+2}\left\|u_{k}\right\|_{q-p+2, a}^{q-p+2} \\
& =c_{k}+\sum_{n \in \mathbb{Z}} W\left(n, u_{k}(n)\right) \\
& =c_{k}+\sum_{|n| \geq K_{2}} W\left(n, u_{k}(n)\right)+\sum_{n=-K_{2}}^{K_{2}} W\left(n, u_{k}(n)\right) \\
& \geq c_{k}-\frac{1}{p(q-p+2)} \sum_{|n| \geq K_{2}} a(n)\left|u_{k}(n)\right|^{q-p+2}-\sum_{n=-K_{2}}^{K_{2}}\left|W\left(n, u_{k}(n)\right)\right| \\
& \geq c_{k}-\frac{1}{p(q-p+2)}\left\|u_{k}\right\|_{q-p+2, a}^{q-p+2}-\sum_{n=-K_{2}}^{K_{2}} \max _{|x| \leq 2 C_{14}}|W(n, x)| .
\end{aligned}
$$

It follows that

$$
c_{k} \leq \frac{1}{p}\left\|\Delta u_{k}\right\|_{l^{p}}^{p}+\frac{p+1}{q-p+2}\left\|u_{k}\right\|_{q-p+2, a}^{q-p+2}+\sum_{n=-K_{2}}^{K_{2}} \max _{|x| \leq 2 C_{14}}|W(n, x)|<+\infty .
$$


This contradicts the fact that $\left\{c_{k}\right\}_{k=1}^{\infty}$ is unbounded, and so $\left\{\left\|u_{k}\right\|\right\}$ is unbounded. The proof is complete.

Proof of Theorem 1.4 In view of the proofs of Theorem 1.2 and Theorem 1.3, the conclusion of Theorem 1.4 holds. The proof is complete.

\section{Examples}

Example 4.1 Consider the following system:

$$
\Delta\left(|\Delta u(n-1)|^{2} \Delta u(n-1)\right)-a(n)|u(n)|^{3} u(n)+\nabla W(n, u(n))=0, \quad \text { a.e. } n \in \mathbb{Z},
$$

where $p=4, q=7, n \in \mathbb{Z}, u \in \mathbb{R}^{N}, a: \mathbb{Z} \rightarrow(0, \infty)$, and $a$ satisfies (A). Let

$$
W(n, x)=a(n)\left(\sum_{i=1}^{m_{1}} a_{i}|x|^{\mu_{i}}-\sum_{j=1}^{m_{2}} b_{j}|x|^{e_{j}}\right),
$$

where $\mu_{1}>\mu_{2}>\cdots>\mu_{m_{1}}>\varrho_{1}>\varrho_{2}>\cdots>\varrho_{m_{2}}>5, a_{i}, b_{j}>0, i=1, \ldots, m_{1}, j=1, \ldots, m_{2}$. Let

$$
W_{1}(n, x)=a(n) \sum_{i=1}^{m_{1}} a_{i}|x|^{\mu_{i}}, \quad W_{2}(n, x)=a(n) \sum_{j=1}^{m_{2}} b_{j}|x|^{\varrho_{j}} .
$$

Then it is easy to check that all the conditions of Theorem 1.3 are satisfied with $\mu=\mu_{m_{1}}$ and $\varrho=\varrho_{1}$. Hence, problem (4.1) has an unbounded sequence of homoclinic solutions.

Example 4.2 Consider the following system:

$$
\Delta\left(|\Delta u(n-1)|^{-1 / 2} \Delta u(n-1)\right)-a(n)|u(n)|^{3 / 2} u(n)+\nabla W(n, u(n))=0, \quad \text { a.e. } n \in \mathbb{Z},
$$

where $p=3 / 2, q=3, n \in \mathbb{Z}, u \in \mathbb{R}^{N}, a: \mathbb{Z} \rightarrow(0, \infty)$ and $a$ satisfies (A). Let

$$
W(n, x)=a(n)\left[a_{1}|x|^{\mu_{1}}+a_{2}|x|^{\mu_{2}}-b_{1}(\sin n)|x|^{\varrho_{1}}-b_{2}|x|^{\varrho_{2}}\right],
$$

where $\mu_{1}>\mu_{2}>\varrho_{1}>\varrho_{2}>7 / 2, a_{1}, a_{2}>0, b_{1}, b_{2}>0$. Let

$$
W_{1}(n, x)=a(n)\left(a_{1}|x|^{\mu_{1}}+a_{2}|x|^{\mu_{2}}\right), \quad W_{2}(n, x)=a(n)\left[b_{1}(\sin n)|x|^{\varrho_{1}}+b_{2}|x|^{\varrho_{2}}\right] .
$$

Then it is easy to check that all the conditions of Theorem 1.4 are satisfied with $\mu=\mu_{2}$ and $\varrho=\varrho_{1}$. Hence, by Theorem 1.4, problem (4.2) has an unbounded sequence of homoclinic solutions. 


\section{Acknowledgements}

This work was supported by the NNSF of China (No. 11301108), Guangxi Natural Science Foundation

(No. 2013GXNSFBA019004) and the Scientific Research Foundation of Guangxi Education Office (No. 201203YB093).

Received: 20 August 2014 Accepted: 10 November 2014 Published: 25 Nov 2014

\section{References}

1. Shi, XB, Zhang, QF, Zhang, QM: Existence of homoclinic orbits for a class of $p$-Laplacian systems in a weighted Sobolev space. Bound. Value Probl. 2013, 137 (2013)

2. Salvatore, A: Homoclinic orbits for a class of strictly convex Hamiltonian systems. Dyn. Syst. Appl. 6, 153-164 (1997)

3. Salvatore, A: On the existence of homoclinic orbits for a second-order Hamiltonian system. Differ. Integral Equ. 10(2), 381-392 (1997)

4. Chen, $\mathrm{P}$, Tang, XH: New existence of homoclinic orbits for a second-order Hamiltonian system. Comput. Math. Appl. 62(1), 131-141 (2011)

5. Poincaré, H: Les méthodes nouvelles de la mécanique céleste. Gauthier-Villars, Paris (1897-1899)

6. Agarwal, RP, Popenda, J: Periodic solution of first order linear difference equations. Math. Comput. Model. 22(1), 11-19 (1995)

7. Agarwal, RP, Perera, K, O'Regan, D: Multiple positive solutions of singular discrete $p$-Laplacian problems via variational methods. Adv. Differ. Equ. 2005(2), 93-99 (2005)

8. Guo, ZM, Yu, JS: The existence of periodic and subharmonic solutions for second order superlinear difference equations. Sci. China Ser. A 46, 506-513 (2003)

9. Guo, ZM, Yu, JS: Periodic and subharmonic solutions for superquadratic discrete Hamiltonian systems. Nonlinear Anal. 55, 969-983 (2003)

10. Guo, ZM, Yu, JS: The existence of periodic and subharmonic solutions of subquadratic second order difference equations. J. Lond. Math. Soc. 68, 419-430 (2003)

11. Liang, $\mathrm{HH}$, Weng, PX: Existence and multiple solutions for a second order difference boundary value problem via critical point theory. J. Math. Anal. Appl. 326, 511-520 (2007)

12. Rodriguez, J, Etheridge, DL: Periodic solutions of nonlinear second order difference equations. Adv. Differ. Equ. 2005(2), 173-192 (2005)

13. Xue, YF, Tang, CL: Existence of a periodic solution for subquadratic second-order discrete Hamiltonian system. Nonlinear Anal. 67, 2072-2080 (2007)

14. Yu, JS, Guo, ZM, Zou, X: Positive periodic solutions of second order self-adjoint difference equations. J. Lond. Math. Soc. 71(2), 146-160 (2005)

15. Yu, JS, Long, YH, Guo, ZM: Subharmonic solutions with prescribed minimal period of a discrete forced pendulum equation. J. Dyn. Differ. Equ. 16, 575-586 (2004)

16. Yu, JS, Deng, XQ, Guo, ZM: Periodic solutions of a discrete Hamiltonian system with a change of sign in the potential. J. Math. Anal. Appl. 324, 1140-1151 (2006)

17. Zhou, Z, Yu, JS, Guo, ZM: Periodic solutions of higher-dimensional discrete systems. Proc. R. Soc. Edinb. A 134 1013-1022 (2004)

18. Wang, Y, Zhang, XY: Multiple periodic solutions for a class of nonlinear difference systems with classical or bounded $\left(\phi_{1}, \phi_{2}\right)$-Laplacian. Adv. Differ. Equ. 2014, 218 (2014)

19. Zhang, XY: Notes on periodic solutions for a nonlinear discrete system involving the $p$-Laplacian. Bull. Malays. Math Soc. 37(2), 499-509 (2014)

20. Zhang, $\mathrm{XY}$, Tang, $\mathrm{XH}$ : Non-constant periodic solutions for second order Hamiltonian system involving the $p$-Laplacian. Adv. Nonlinear Stud. 13(4), 945-964 (2013)

21. Zhang, $X Y$, Tang, $X H$ : A note on the minimal periodic solutions of nonconvex superlinear Hamiltonian system. Appl. Math. Comput. 219, 7586-7590 (2013)

22. Zhang, XY, Tang, XH: Some united existence results of periodic solutions for non-quadratic second order Hamiltonian systems. Commun. Pure Appl. Anal. 13, 75-95 (2014)

23. Zhang, QF, Tang, XH: Existence of homoclinic orbits for a class of asymptotically $p$-linear difference systems with p-Laplacian. Abstr. Appl. Anal. 2011, 351562 (2011)

24. Chen, $\mathrm{P}$, Tang, XH: Existence of infinitely many homoclinic orbits for fourth-order difference systems containing both advance and retardation. Appl. Math. Comput. 217, 4408-4415 (2011)

25. He, XF, Chen, P: Homoclinic solutions for second order discrete $p$-Laplacian systems. Adv. Differ. Equ. 2011, 57 (2011)

26. Chen, $\mathrm{P}$, Tang, XH: Existence of homoclinic solutions for a class of nonlinear difference equations. Adv. Differ. Equ. 2010, 470375 (2010)

27. Chen, $\mathrm{P}$, Tang, XH: Existence of homoclinic orbits for 2nth-order nonlinear difference equations containing both many advances and retardations. J. Math. Anal. Appl. 381, 485-505 (2011)

28. Fang, H, Zhao, DP: Existence of nontrivial homoclinic orbits for fourth-order difference equations. Appl. Math. Comput. 214, 163-170 (2009)

29. Lin, X, Tang, XH: Existence of infinitely many homoclinic orbits in discrete Hamiltonian systems. J. Math. Anal. Appl. 373(1), 59-72 (2011)

30. Ma, M, Guo, ZM: Homoclinic orbits and subharmonics for nonlinear second order difference equations. Nonlinear Anal. 67, 1737-1745 (2007)

31. Ma, M, Guo, ZM: Homoclinic orbits for second order self-adjoint difference equations. J. Math. Anal. Appl. 323(1), 513-521 (2006)

32. Yu, JS, Shi, HP, Guo, ZM: Homoclinic orbits for nonlinear difference equations containing both advance and retardation. J. Math. Anal. Appl. 352, 799-806 (2009)

33. Chen, P, Tang, XH: Infinitely many homoclinic solutions for the second-order discrete p-Laplacian systems. Bull. Belg. Math. Soc. Simon Stevin 20, 193-212 (2013)

34. Zhang, XY: Homoclinic orbits for a class of $p$-Laplacian systems with periodic assumption. Electron. J. Qual. Theory Differ. Equ. 2013, 67 (2013) 
35. Tang, XH, Lin, XY: Existence of infinitely many homoclinic orbits in Hamiltonian systems. Proc. R. Soc. Edinb., Sect. A $141,1103-1119(2011)$

36. Benci, V, Fortunato, D: Weighted Sobolev space and the nonlinear Dirichlet problem in unbounded domains. Ann. Mat. Pura Appl. 121, 319-336 (1979)

37. Rabinowitz, PH: Minimax Methods in Critical Point Theory with Applications to Differential Equations. CBMS Regional Conf. Ser. in Math., vol. 65. Am. Math. Soc., Providence (1986)

10.1186/1687-1847-2014-291

Cite this article as: Zhang: Existence of homoclinic solutions for a class of difference systems involving $p$-Laplacian. Advances in Difference Equations 2014, 2014:291

Submit your manuscript to a SpringerOpen ${ }^{\circ}$ journal and benefit from:

- Convenient online submission

Rigorous peer review

- Immediate publication on acceptance

Open access: articles freely available online

- High visibility within the field

- Retaining the copyright to your article

Submit your next manuscript at $\boldsymbol{s p r i n g e r o p e n . c o m ~}$ 